Hypoxia

\section{Injury and repair in developing brain}

\section{F M Vaccarino, L R Ment}

\section{Animal models show that developmental compensatory mechanisms may promote neural and functional plasticity}

$\mathrm{P}$ reterm birth results in considerable disability, yet several reports suggest recovery from injury in developing brain. Developmental compensatory mechanisms may promote neural and functional plasticity, and numerous experimental studies have documented the brain's ability to engage in regenerative mechanisms to potentially replace injured cells. We review available evidence for recovery from injury in models for the preterm brain and offer hypotheses for targeting time dependent molecular and cellular repair mechanisms that have been recently gathered from animal studies. A better understanding of these adaptive cellular and molecular mechanisms will help clinicians apply knowledge derived from animal models to clinical situations.

\section{NEWBORN RODENT AS A GOOD MODEL FOR PRETERM BRAIN}

The many neurodevelopmental handicaps that very low birthweight infants experience suggest that preterm birth disrupts the genetically programmed pattern of brain genesis. To develop a clinically relevant model of the effect of preterm birth on developing brain, one must use an animal model that shows that the injury imposed results in neuropathological changes similar to those found in preterm infants and correlate these changes with behavioural outcomes. As in the preterm infant at the end of the second trimester, neuronal generation in the newborn rodent is complete in most regions, axonal and dendritic branching is robust, and synaptogenesis is just beginning. ${ }^{1}$

Review of the literature suggests that oxygen deprivation is a major cause of neurodevelopmental disability in preterm infants. ${ }^{2}$ Although intraventricular haemorrhage, periventricular leucomalacia, and ventriculomegaly are the most commonly recognised and best studied of these circulatory disturbances, ${ }^{2}$ hypoxia is particularly prevalent among very low birthweight infants and is a common denominator of these abnormalities. ${ }^{3}$
Models of both hypoxia-ischaemia and hypoxia have been studied in newborn rodents, and the former results in focal injury to developing brain. ${ }^{4}$ In contrast, the exposure of young animals to hypoxia mimics global injury to the preterm brain. ${ }^{5}$ Decreases in brain weight, cortical volumes, and neuronal size as well as ventriculomegaly have been reported in neonatal rats and mice exposed to periods of hypoxic injury. ${ }^{67}$ Dendritic spine development is impaired after hypoxia in newborn rodents, ${ }^{8}$ and a recent molecular analysis of the effect of chronic sublethal hypoxia on developing mouse brain showed disruption in those genes subserving synaptogenesis. ${ }^{9}$ Finally, animals exposed to chronic hypoxia experience hyperactivity and long term impairment of spatial memory abilities. Taken together, these data suggest that chronic hypoxia results in significant alterations in brain development and maturation in the newborn rodent model similar to those found in very low birthweight preterm infants.

\section{POSSIBLE CONTRIBUTION OF POSTNATAL NEUROGENESIS TO REORGANISATION OF BRAIN AFTER INJURY}

Multiple animal studies have shown that the brain can reorganise patterns of connections to recover from or compensate for injury during development, ${ }^{11}$ and this phenomenon of plasticity has been variously attributed to increases in neurogenesis and synaptogenesis or to the reorganisation of existing circuitry. ${ }^{12}{ }^{13}$ Although it seems plausible that neonates can reorganise patterns of connections during the time when these are still being refined, whether or not recovery from injury involves actual regeneration of nerve cells and reconstruction of circuitry is controversial.

Further, it has been known for many years that certain regions of the postnatal and adult brain contain neural stem cells able to undergo constitutive neurogenesis, $^{14}$ but only relatively recently has this phenomenon been shown in a wide range of mammalian species, including man. ${ }^{15}$ Although neural stem cells are present throughout the brain, only those in the forebrain subventricular zone (SVZ) and the subgranular layer of the dentate gyrus appear to undergo neurogenesis in vivo. These two regions provide neurones for the olfactory bulb and the dentate gyrus respectively. In important recent experiments, newly generated hippocampal granule cells have been shown to integrate themselves into pre-existing circuitry, become electrically active, and form synaptic connections. ${ }^{16} 17$

Postnatal neurogenesis is influenced by both the external and internal environment. Hippocampal neurogenesis declines with age and is suppressed by stress. ${ }^{18}{ }^{19}$ In contrast, the proliferation of hippocampal progenitors is enhanced by oestrogens and by exercise, and the survival of newly born neurones is promoted by an enriched environment. ${ }^{2021}$ The increase in neuronal survival resulting from environmental stimulation-that is, "early intervention"-protects neurones from injury and is possibly due to increased concentrations of neurotrophins in the hippocampus. ${ }^{22}$ As environmental enrichment improves spatial memory in animal models, enhanced neurogenesis and neuronal survival in the dentate gyrus may increase the ability of an animal to learn new information.

Neural stem cells of the postnatal SVZs and the dentate gyrus may also respond to a variety of noxious environmental perturbations. In adult mice, stem cells can reconstitute the whole SVZ even after more than $90 \%$ destruction of this region. ${ }^{23}$ These progenitors give rise to cortical pyramidal neurones if there is massive apoptosis in the cerebral cortex ${ }^{24}$ or to hippocampal pyramidal neurones and striatal neurones after experimental stroke in adult rats. ${ }^{25}$ The newly generated neurones are targeted to the injured site, suggesting that neurogenesis is regulated by local changes in gene expression after injury. ${ }^{26}$ Finally, Nakatomi et al ${ }^{27}$ have described extensive regeneration (up to $40 \%$ ) of the hippocampal CAl pyramidal layer after ischaemia in adult rats, but only after infusions of basic fibroblast growth factor (FGF) and epidermal growth factor in the cerebral ventricles. As this degree of regeneration was accompanied by substantial behavioural recovery, ${ }^{27}$ elucidation of those mechanisms that mediate neural regenerative events is critical for the development of therapeutic strategies for injured brains.

Neural stem cells contain glial fibrillary acidic protein, an intermediate filament typical of astrocytes. ${ }^{28}$ Astrocytes arise from radial glia, the

Abbreviations: FGF, fibroblast growth factor; SVZ, subventricular zone 


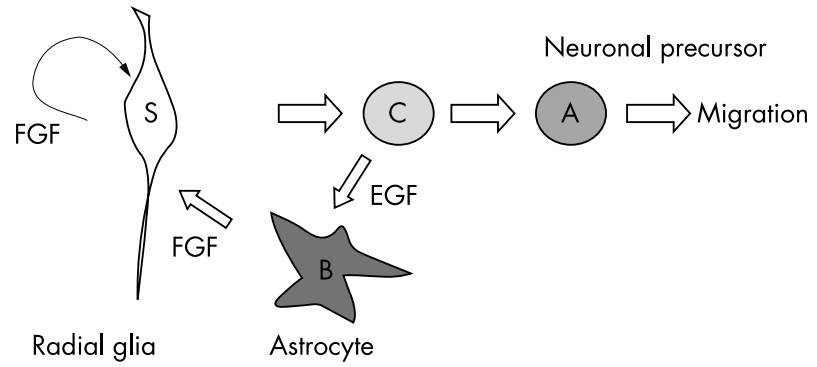

Figure 1 Cells of the astrocyte lineage give rise to neurones in the postnatal subventricular zone (SVZ). In the normal SVZ, there is a steady state equilibrium in which stem cells (S) generate transit amplifying cells (C cells) and neuronal progenitor cells (A cells). C cells also generate astrocytes ( $B$ cells), and this can be increased by exogenous epidermal growth factor (EGF). Astrocytes are normally quiescent. The $C$ and $A$ cells divide with a cell cycle of $13 \mathrm{~h}$. Stem cells, estimated to be about $1 \%$ of the rapidly dividing cells, may lineally derive from astrocytes through a dedifferentiation process, a process that may be enhanced by fibroblast growth factor 2 (FGF2).

mitotically active embryonic progenitor cells that normally form the scaffolding of the developing neuroepithelium. Stretching an apical and a basal process between the ventricular and the pial layers, these cells generate cortical neurones during embryogenesis. After corticogenesis is finished, the radial glia gradually retract their ventricular process, transforming into multipolar astrocytes.

\section{RADIAL GLIA MAY BE CRITICAL FOR RECOVERY FROM INJURY IN DEVELOPING BRAIN}

Recent data have suggested that recovery from injury in the preterm brain may involve the reactivation of radial glia in the germinal layers. ${ }^{29}$ In the days after acute or chronic hypoxic insult, there is increased cell proliferation in both the SVZ and the dentate gyrus, and the "reactive" cells that divide after perinatal hypoxia appear to be phenotypically a form of radial glia. ${ }^{29}$

This abundance of radial glia may be secondary to the increased proliferation of pre-existing radial glia after injury and/or to a reversion of their "involution" into astrocytes. Hence, after brain insults, astrocytes may be able to "rejuvenate" and revert to radial glia, which in turn may generate neurones as shown in fig 1 .

Radial glia express FGF receptors, and several studies in vitro suggest that FGF2 is necessary for the proliferative expansion of these progenitors. ${ }^{30}$ FGF2 may also be important for regenerative phenomena in the postnatal brain. FGF2 concentrations are increased in the recovery phase after neonatal hypoxia, as is the expression of FGF receptor 1 in the SVZ. ${ }^{29}$ Of note, FGF2 message and protein are increased after hypoxia/ischaemia in the adult brain, and adult Fgf2 knockout mice are unable to mount a regenerative response in the hippocampus after hypoxia/ischaemia. Although these results suggest that an increase in FGF2 expression may promote recovery after insults in the adult rodent brain, the significance of the FGF signalling pathway for functional recovery in the neonatal period remains to be elucidated.

In addition, genetic and environmental factors that influence neurogenesis, such as the secretion of growth factors and rodent "early intervention", most certainly affect a variety of growth processes. These include fibre sprouting and synaptogenesis. Thus it will be essential to discriminate from the myriad of changing events those that are critical to direct functional recovery. Candidate genes that potentially play a role in these phenomena are those for growth factors, their receptors, and intracellular transduction events, those that regulate apoptosis, and those neural stem cell transcription factors that regulate lineage determination. Transgenic mice lacking or overexpressing these molecules and exposed to chronic sublethal hypoxia may represent good models for the adaptive mechanisms of developing preterm brain.

Recent clinical studies suggest improvement in some measures of cognitive function in preterm infants across time. Modelling chronic sublethal hypoxia in neonatal rats and mice recapitulates the type of damage that is present in premature infants and in other conditions of chronic neonatal hypoxia. These models may be useful in testing mechanisms of recovery and potential therapeutic strategies. Primitive glial cells such as radial glia may be able to generate new cells for brain repair under conditions in which mature cells are dying, raising hopes that the postnatal brain may be able to support neurogenic programmes after injury. Future research is needed to shed light on the mechanisms that promote the proliferation of these cells, their differentiation into neurones and glia, and their proper integration into functional neuronal circuitry.

Arch Dis Child Fetal Neonatal Ed 2004;89:F190-F192.

doi: 10.1136/adc.2003.043661

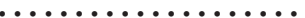

Authors' affiliations

F M Vaccarino, Child Study Center and Department of Neurobiology, Yale University School of Medicine, New Haven, CT, USA L R Ment, Departments of Pediatrics and Neurology, Yale University School of Medicine

Correspondence to: Dr Ment, Department of Pediatrics, Yale University School of Medicine, 333 Cedar Street, New Haven, CT 06511, USA; Laura.ment@yale.edu

This work was supported by NIH NS 35476, NS 27116, MH 49351, NS 37709, and NSF IBN-0083104.

\section{REFERENCES}

1 Dobbing J. Undernutrition and the developing brain: the use of animal models to elucidate the human problem. Psychiatr Neurol Neurochir 1971;74:433-42.

2 Volpe JJ. Perinatal brain injury: from pathogenesis to neuroprotection. Ment Retard Dev Disabil Res Rev 2001 ;7:56-64.

3 Hack M, Wright LL, Shankaran S, et al. Very-lowbirth-weight outcomes of the National Institute of Child Health and Human Development Neonatal Network, November 1989 to October 1990. Am J Obstet Gynecol 1995; 172:457-64.

4 Vexler ZS, Ferriero DM. Molecular and biochemical mechanisms of perinatal brain injury. Semin Neonatol 2001:6:99-108.

5 Back SA, Volpe JJ. Cellular and molecular pathogenesis of periventricular white matter injury. Ment Retard Dev Disabil Res Rev 1997.3.96-107.

6 Laroia N, McBride L, Baggs R, et al. Dextromethorphan ameliorates effects of neonatal hypoxia on brain morphology and seizure threshold in rats. Dev Brain Res 1996;100:29-34.

7 Ment LR, Schwartz M, Makuch RW, et al. Association of chronic sublethal hypoxia with ventriculomegaly in the developing rat brain. Dev Brain Res 1998;111:197-203.

8 Nyakas C, Buwalda B, Luiten P. Hypoxia and brain development. Prog Neurobiol 1996;49:1-51

9 Curristin SM, Cao A, Stewart WB, et al. Disrupted synaptic development in the hypoxic newborn brian. Proc Natl Acad Sci USA 2002;99:15729-34

10 Kitagawa K, Matsumotor M, Hori M. Protective and regenerative response endogenously induced in the ischemic brain. Can J Physiol Pharmacol 2001;79:262-5.

11 Perretto P, Merighi A, Fasolo A, et al. The subependymal layer in rodents: a site of structural plasticity and cell migration in the adult mammalian brain. Brain Res Bull 1999;49:221-43.

12 Alvarez-Buylla A, Herrera DG, Wichterle H. The subventricular zone: source of neuronal precursors for brain repair. Prog Brain Res 2000; 127:1-11.

13 Hitoshi S, Tropepe V, Ekker M, et al. Neural stem cell lineages are regionally specified, but not committed, within distinct compartments of the developing brain. Development 2002; 129:233-44.

14 Altman J, Das GD. Autoradiographic and histological evidence of postnatal hippocampal neurogenesis in rats. J Comp Neurol 1965; 124:319-35.

15 Eriksson PS, Perfilieva E, Bjork-Eriksson T, et al. Neurogenesis in the adult human hippocampus [comment]. Nat Med 1998:4:1313-17.

16 Markakis EA, Gage FH. Adult-generated neurons in the dentate gyrus send axonal projections to 
field CA3 and are surrounded by synaptic vescicles. J Comp Neurol 1999;406:449-60.

17 Song HJ, Stevens CF, Gage FH. Neural stem cells from adult hippocampus develop essential properties of functional CNS neurons. $\mathrm{Na}$ Neurosci 2002; 5:438-45

18 Gould E, Tanapat P, McEwen BS, et al. Proliferation of granule cell precursors in the dentate gyrus of adult monkeys is diminished by stress. Proc Natl Acad Sci USA 1998;95:3168-71.

19 Kempermann G, Kuhn HG, Gage FH Experience-induced neurogenesis in the senescen dentate gyrus. J Neurosci 1998;18:3206-12.

20 Tanapat AJ, Hastings NB, Reeves AJ, et al. Estrogen stimulates the proliferation of granule cell precursors in the dentate gyrus of adult female rats. Neuroscience Abstracts 1998;24:796-8.

21 van Praag H, Kempermann G, Gage FH. Neura consequences of environmental enrichment. Nat Rev Neurosci 2000;1:191-8.
22 Young D, Lawlor PA, Leone $P$, et al Environmental enrichment inhibits spontaneous apoptosis, prevents seizures and is neuroprotective. Nat Med 1999;5:448-53.

23 Doetsch F, Garcia-Verdugo JM, Alvarez-Buylla A. Regeneration of a germinal layer in the adult mammalian brain. Proc Natl Acad Sci USA 1999;96:11619-24.

24 Magavi SS, Macklis JD. Induction of neuronal type-specific neurogenesis in the cerebral cortex of adult mice: manipulation of neural precursors in situ. Dev Brain Res 2002;134:57-76.

25 Arvidsson A, Collin T, Kirik D, et al. Neuronal replacement from endogenous precursors in the adult brain after stroke. Nat Med 2002;8:963-70.

26 Wang Y, Sheen VL, Macklis JD. Cortical interneurons upregulate neurotrophins in vivo in response to targeted apoptotic degeneration of neighboring pyramidal neurons. Exp Neurol 1998; 154:389-402.
27 Nakatomi H, Kuriu T, Okabe S, et al. Regeneration of hippocampal pyramidal neurons after ischemic brain injury by recruitment of endogenous neural precursors. Cell 2002; 110:429-41.

28 Doetsch F, Caille I, Lim DA, et al. Subventricular zone astrocytes are neural stem cells in the adult mammalian brain. Cell 1999;97:703-16.

29 Ganat Y, Soni S, Chacon M, et al. Chronic hypoxia up-regulates fibroblast growth factor ligands in the perinatal brian and induces fibroblast growth factor-responsive radial glial cells in the sub-ependymal zone. Neuroscience 2002; 112:977-91.

30 Vaccarino FM, Schwartz ML, Hartigan D, et al. Effect of basic fibroblast growth factor on the genesis of excitatory and inhibitory neurons in primary cultures of cells from the mammalian telencephalon. Cereb Cortex 1995; 1:64-78. 